\title{
Power and temperature dependence of low frequency noise in AIGaN/GaN transmission line model structures
}

\author{
S. A. Vitusevich, ${ }^{\text {a) }}$ S. V. Danylyuk, and N. Klein \\ Institut für Schichten und Grenzfä̈chen and CNI-Center of Nanoelectronic Systems for Information \\ Technology, Forschungszentrum Jülich, 52425 Jülich, Germany \\ M. V. Petrychuk \\ Taras Shevchenko National University, Kiev 01033, Ukraine \\ A. E. Belyaev \\ V. Lashkaryov Institute of Semiconductor Physics, NASU, 03028 Kiev, Ukraine
}

(Received 25 February 2004; accepted 18 August 2004)

\begin{abstract}
The low-frequency noise in $\mathrm{AlGaN} / \mathrm{GaN}$ transmission line model structures has been investigated for a wide range of ambient temperatures and dissipated powers. A deviation of low-frequency noise from a $1 / f$ dependence has been observed upon increasing the applied voltage. The effect correlates with the nonlinearity of current-voltage characteristics (CVC). The concept of nonequilibrium $1 / f$ noise has been introduced to explain $1 / f$ noise enhancement. A qualitative model connecting non-equilibrium noise with nonlinearity of the CVC via a common origin-nonuniform overheating of the structures - has been suggested. Furthermore, deviations of equilibrium noise from the $1 / f$ law at low temperatures have been observed and studied. (C) 2004 American Institute of Physics. [DOI: 10.1063/1.1805719]
\end{abstract}

\section{INTRODUCTION}

The recognition of $\mathrm{AlGaN} / \mathrm{GaN}$ structures with conducting two-dimensional (2D) electron gas has considerably increased during recent years due to a number of unique properties such as wide bandgap, polarization induced doping, and high breakdown fields of the Schottky barriers to the AlGaN. These properties allow the power handling capability of nitride-based devices, e.g., high electron mobility transistors (HEMTs), to be increased in comparison with conventional GaAs-based devices. ${ }^{1}$ At the same time, the lowfrequency noise level in GaN-based transistors does not exceed that in GaAs-based HEMTs. ${ }^{2}$

High operation power of these devices leads to a significant self-heating effect and, consequently, a change in their working parameters. In HEMTs the self-heating effects reduce the 2D electron mobility and cause sublinearity in the current-voltage characteristics (CVC) of the device. ${ }^{3}$ Moreover, the nonuniformity of the electric field applied to the channel due to lateral redistribution of charge on the surface or in the barrier also results in an increased channel resistance. ${ }^{4}$

To investigate the above mentioned effects, transmission line model (TLM) structures are most suitable. They represent a HEMT structure with conducting channels of different length without nonuniformities in the electrical field introduced by the gate electrode. In previous investigations, nonlinearity effects of current-voltage characteristics in TLMstructures have been studied. ${ }^{5-7}$ In the low-voltage region the CVC follows Ohm's law. By increasing the voltage applied the dependence becomes sublinear, followed by a saturation

\footnotetext{
a) Author to whom correspondence should be addressed; on leave from Institute of Semiconductor Physics, NASU, 03028 Kiev, Ukraine; electronic mail: s.vitusevich@fz-juelich.de
}

region, and sometimes even a decrease in current can be observed. The latter is not typical and gives evidence of nonequilibrium processes in the $2 \mathrm{D}$ electron gas in TLM structures as well as in similar HEMT structures. It should be noted that among a huge number of publications on the investigation of low-frequency noise in nitride structures, there are none with an analysis of nonequilibrium noise effects. Noise properties of these structures have been investigated with the assumption (explicit or implicit) of equilibrium noise.

In general, the equilibrium noise corresponds to fluctuations of the resistance, which exist in a sample even without current flow through the structure. ${ }^{8,9}$ The small currents just help to reveal these fluctuations ${ }^{10,11}$ and for a homogeneous sample the following relations should be used at a certain frequency:

$$
\frac{S_{I}}{I^{2}} \sim \frac{S_{R}}{R^{2}}=\text { const }
$$

where $S_{I}$ and $S_{R}$ are the spectral density of current and resistance fluctuations, respectively, $I$ is the current, and $R$ is the resistance of the sample.

With increasing applied voltage the intrinsic state of the system changes due to current flow and nonequilibrium noise dominates in the system. ${ }^{12,13}$ Nonequilibrium noise can be distinguished not only by deviations from linearity in dependence $S_{I} \sim I^{2}{ }^{14}$ but also by changes in the shape of the spectra. This occurs in the case when the system is no longer in a state of thermal equilibrium and, as a result, the noise response deviates from $1 / f$ dependence. We observed noise of this type in TLM structures under certain conditions. ${ }^{15}$ Probably, fingerprints of nonequilibrium noise have also been revealed in HEMT structures. Nonequilibrium noise described in Ref. 15 appears as an increase of the $S_{I} / I^{2}$ value against 
the original $1 / f$ noise in the low-frequency range of the spectrum. In the frequency region where the transition from equilibrium to nonequilibrium noise occurs the relative noise spectral density $S_{I} / I^{2}$ is proportional to $1 / f^{2}$. Further, the value of $S_{I} / I^{2}$ for nonequilibrium noise rises by increasing the applied voltage and saturates at certain values of the voltage. The frequency of transition from linear to quadratic slope in the $S_{I} / I^{2}=F(\mathrm{f})$ dependence also increases with applied voltage. In this work we present an analysis of the behavior and origin of equilibrium and nonequilibrium $1 / f$ noise in GaN-based 2D electron gas.

\section{EXPERIMENT}

In our experiment we used gateless TLM devices. A detailed description of samples can be found elsewhere. ${ }^{3} \mathrm{We}$ used samples with channel length of 10, 15, 20, 25, 30, and $35 \mu \mathrm{m}$. The applied voltage was varied in the range from 0.01 to $10 \mathrm{~V}$, the maximal value of measured current did not exceed $112 \mathrm{~mA}$. The noise spectra were measured in the frequency range from $1 \mathrm{~Hz}$ to $100 \mathrm{kHz}$. The measurements at room temperature were carried out without additional heat sink, while the low temperature measurements were performed with an active heat sink with stabilized temperature within a range of temperatures from 70 to $300 \mathrm{~K}$.

\section{RESULTS AND DISCUSSION}

\section{A. Equilibrium noise case}

First the equilibrium noise results will be discussed. In our case the noise level $S_{I} / I^{2}$ obeys Eq. (1) up to several hundred millivolts. In this voltage range we consider the dominant contribution of the equilibrium part in the total noise. According to Fig. 1, there is a typical $1 / f$ noise dependence at room temperature. In some cases generationrecombination excess noise was observed, but the power of the $1 / f$ noise dependence in these cases was also equal to unity. The question concerning the origin of $1 / f$ noise in HEMTs is widely discussed in the literature. ${ }^{16}$ The main discussion topic is the model appropriate for describing the origin of the observed noise. At the moment, two theoretical approaches to describe the $1 / f$ noise are discussed: the McWhorter and Hooge models. The main physical difference between these two cases is the origin of noise sources. McWhorter's model of the noise deals with surface or interface noise sources (fluctuations of concentration, $\Delta n),{ }^{17}$ while Hooge's model takes into consideration the distribution of noise sources in the volume (mobility fluctuations, $\Delta \mu) .{ }^{18}$ The question whether $\Delta n$ or $\Delta \mu$ is responsible for current fluctuations even in the case of equilibrium noise still remains open.

The difference, however, is very difficult to distinguish experimentally and can be determined only for some special cases. For our TLM structures case, as objects of investigation both the McWhorter and Hooge models lead to a $f S_{I} / I^{2} \sim 1 / L$ dependence of normalized noise on the sample length. Such dependence is confirmed by our measurements (see inset of Fig. 1) and is in good agreement with both theories. In the Hooge model, in which the noise is determined by fluctuation of the channel resistance caused by

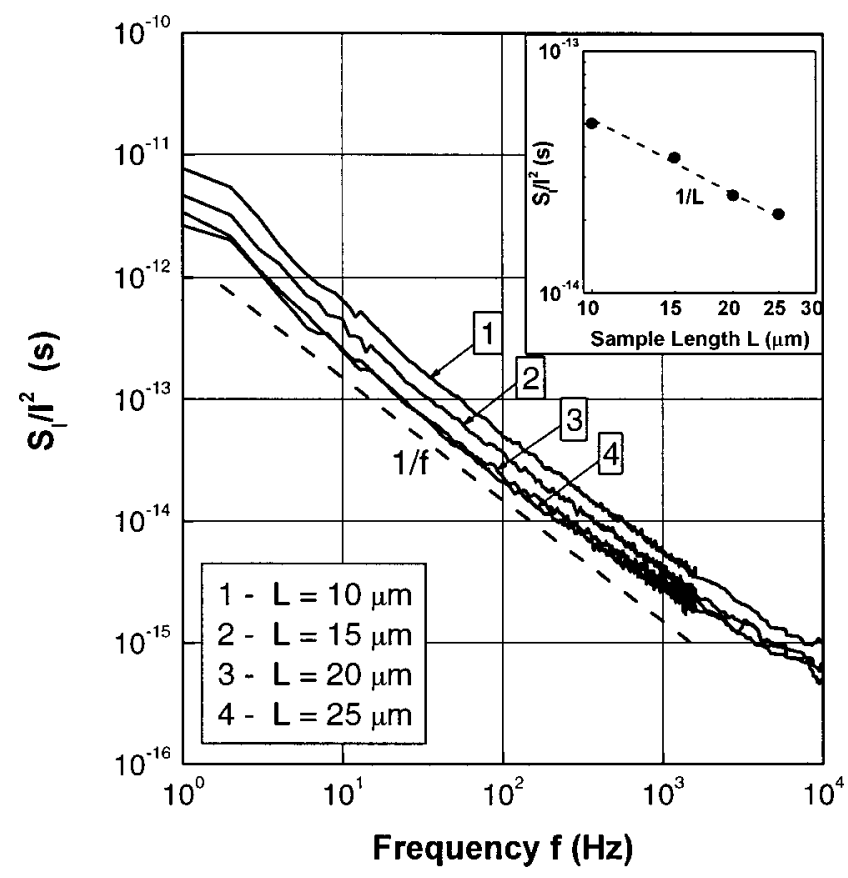

FIG. 1. Frequency spectra of the equilibrium noise for samples with channel length from 10 to $25 \mu \mathrm{m}$ measured at an applied voltage of $100 \mathrm{mV}(T$ $=300 \mathrm{~K}$ ). Upper inset: spectral noise density dependence on channel length measured at $f=100 \mathrm{~Hz}$. Dashed line shows inverse proportional dependence of $L$.

fluctuations of the carrier mobility, the Hooge parameter $\alpha_{H}$ can be estimated. For the spectra presented in Fig. 1 its value was found to be $\alpha_{H}=4 \times 10^{-4}$. This result agrees fairly well with previously reported data of other authors. ${ }^{19}$

On the other hand, deviations from $1 / f$ dependence of equilibrium noise were observed in the spectra of TLM structures at lower temperatures [Figs. 2(a) and 2(b)]. It should be noted that the transformation of the spectra is of a complex nature. Upon the lowering temperature from 300 to $200 \mathrm{~K}$ the level of normalized noise at high frequencies increases considerably, while the low-frequency part of the spectra remains practically unchanged. The transient frequency (with $\gamma \approx 0.8$ in $1 / f$ dependence) corresponds to the frequency between unchanged and changed parts of the spectra. The frequency shifts towards low frequency and exhibits an exponential dependence on temperature with an activation energy of $0.4 \mathrm{eV}$ (Fig. 3). In McWhorter's model the origin of $1 / f$ noise is considered to be caused by uniformly distributed traps in space and energy. ${ }^{20}$ Therefore, any deviation from $1 / f$ dependence can be explained as a result of nonuniform distribution of noise sources. However, the exponential temperature dependence of the frequency shift is not inherent in the McWhorter $1 / f$ noise. Thus, McWhorter's model alone cannot explain the features observed in the spectra with decreasing temperature and should be supplemented to describe the observed features.

It is known, however, that free-energy change in the barrier system caused by lateral transfer from $2 \mathrm{D}$ electron gas to a trap location must be taken into account in the capture and emission processes. ${ }^{21}$ The free-energy change may be of the order of several hundred millivolts. ${ }^{22}$ and this effect usually referred in literature as Coulomb blockade. ${ }^{23}$ In this case 

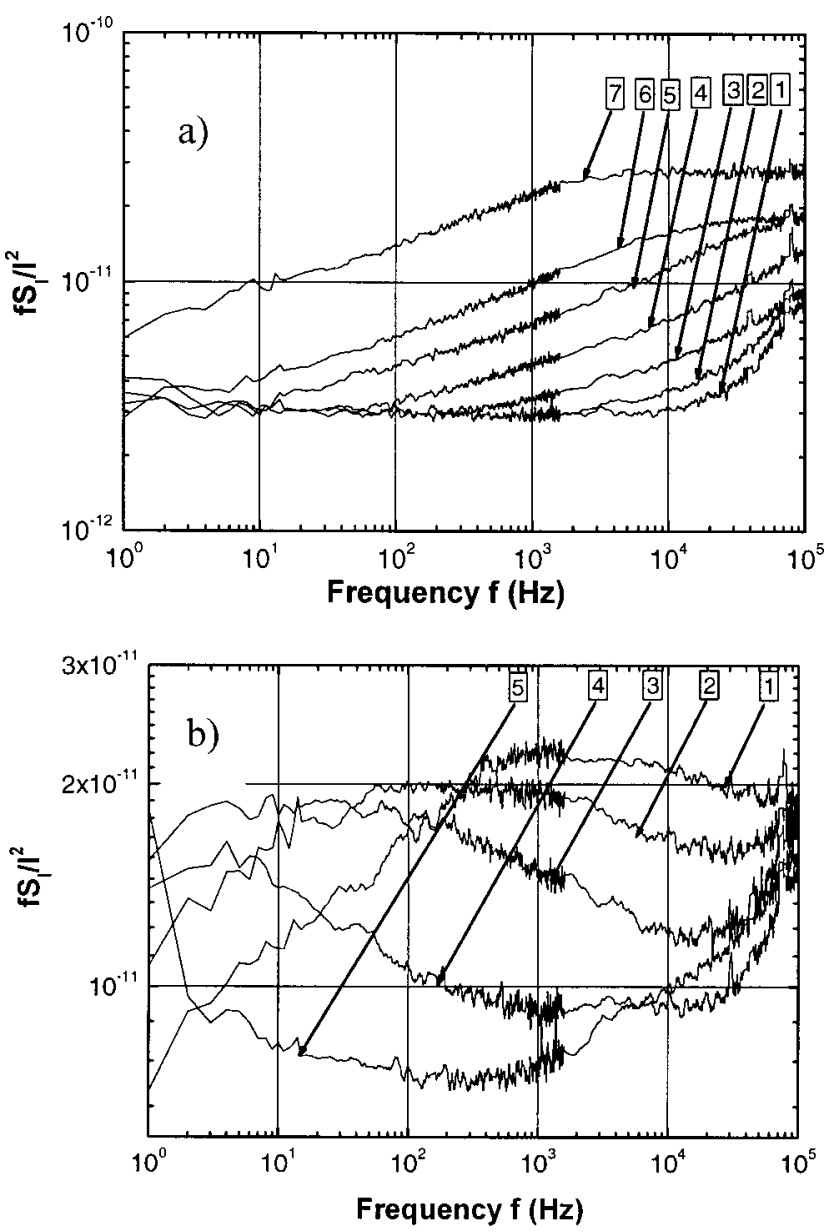

FIG. 2. Normalized spectra of the equilibrium noise measured for sample with channel length of $10 \mu \mathrm{m}$ at $V=500 \mathrm{mV}$. Temperature of copper sample holder was changed in steps of $20 \mathrm{~K}$ : (a) from $T_{0}=300 \mathrm{~K}$ (curve 1) to $T_{0}$ $=180 \mathrm{~K}$ (curve 7 ), (b) from $160 \mathrm{~K}$ (curve 1 ) to $80 \mathrm{~K}$ (curve 5).

additional barrier for electron tunneling from $2 \mathrm{D}$ channel to traps in the barrier appears that strongly affects the capturing time. According to Coulomb blockade theory electron capturing time can be written as formula

$$
\tau_{c}=\frac{1}{C_{n} n_{s}} \exp \left(\frac{\Delta E}{k T}\right),
$$

where $\Delta E$ is Coulomb energy, $C_{n}$ is electron capture constant, and $n_{s}$ is electron density. Thereby even with presence

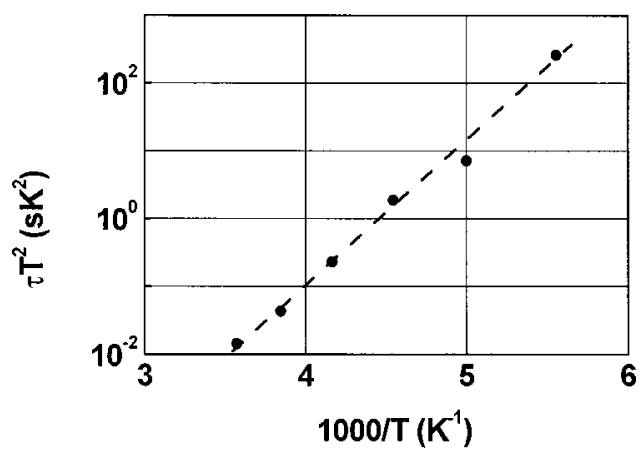

FIG. 3. Inverse temperature dependence of normalized transient time corresponding to the noise spectral density of Fig. 2(a) at the level of $9 \times 10^{-12}$ (Arrhenius plot). Dashed line corresponds to linear fit with the activation energy level of $0.4 \mathrm{eV}$.

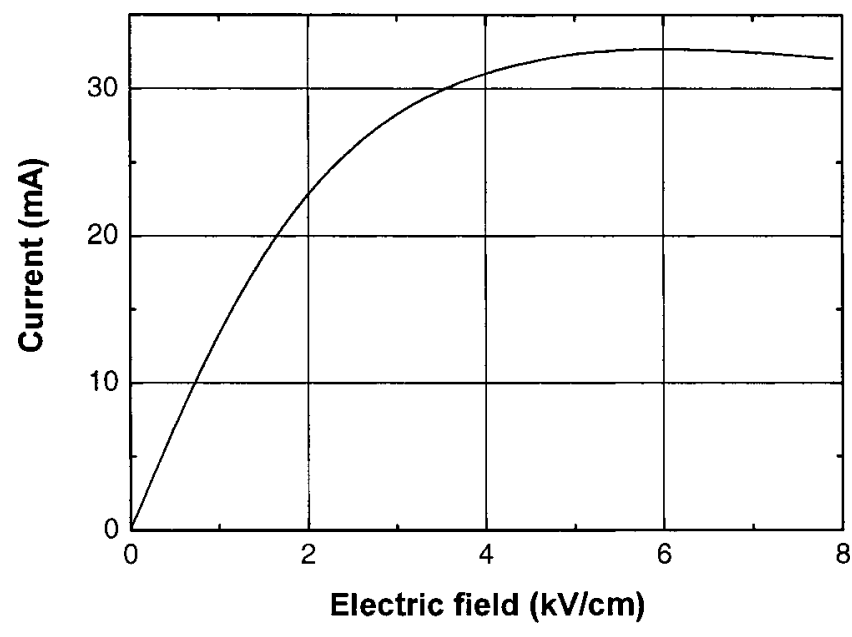

FIG. 4. Current-voltage characteristic for the device with channel length of $25 \mu \mathrm{m}$ and width of $100 \mu \mathrm{m}$ measured at $T=300 \mathrm{~K}$.

of tunneling the electron capturing is thermoactivated process. In our case the value of Coulomb energy is $0.4 \mathrm{eV}$.

\section{B. Nonequilibrium noise and its correlation with equilibrium noise}

The current-voltage characteristics of the samples become strongly nonlinear (Fig. 4) in TLM structures when the applied voltage exceeds $1 \mathrm{~V}$. At these voltages (Fig. 5) a strong deviation from $1 / f$ dependence was observed in noise spectra. This means that noise and transport effects are correlated and we will analyze them from the point of view of nonequilibrium conditions.

The resistance of a sample increases with applied voltage, thus we can write it at each voltage in the following form: ${ }^{14}$

$$
R=R_{0}+\Delta R,
$$

where $R_{0}$ describes the ohmic part of CVC and $\Delta R$ is the deviation from linear dependence on applied voltage components of the total resistance. Since the $I-V$ characteristics of TLM structures are strongly nonlinear (a resistance increases

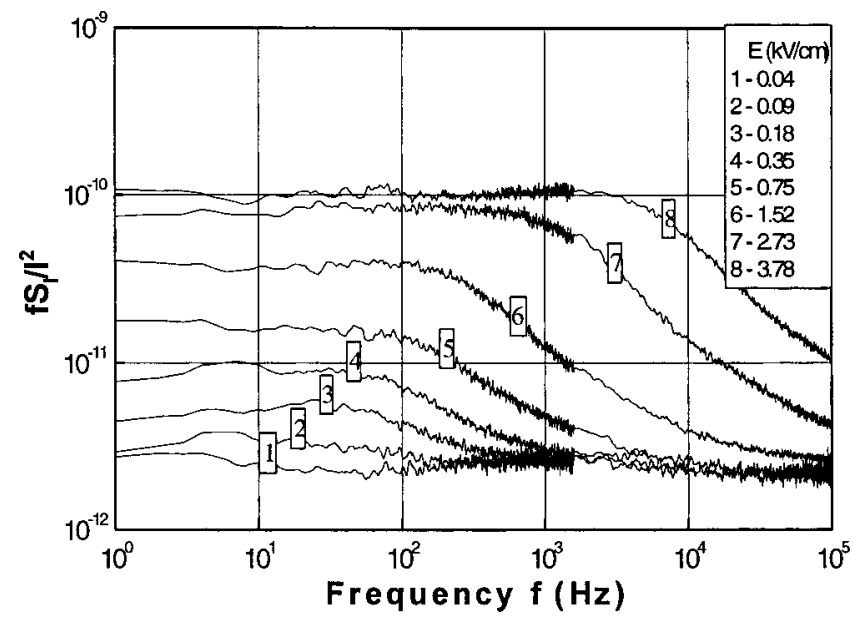

FIG. 5. Spectra of the normalized current noise for different values of the electric field $\mathrm{E}$ measured at $T=300 \mathrm{~K}$ for the device with channel length of $25 \mu \mathrm{m}$ and width of $100 \mu \mathrm{m}$. 


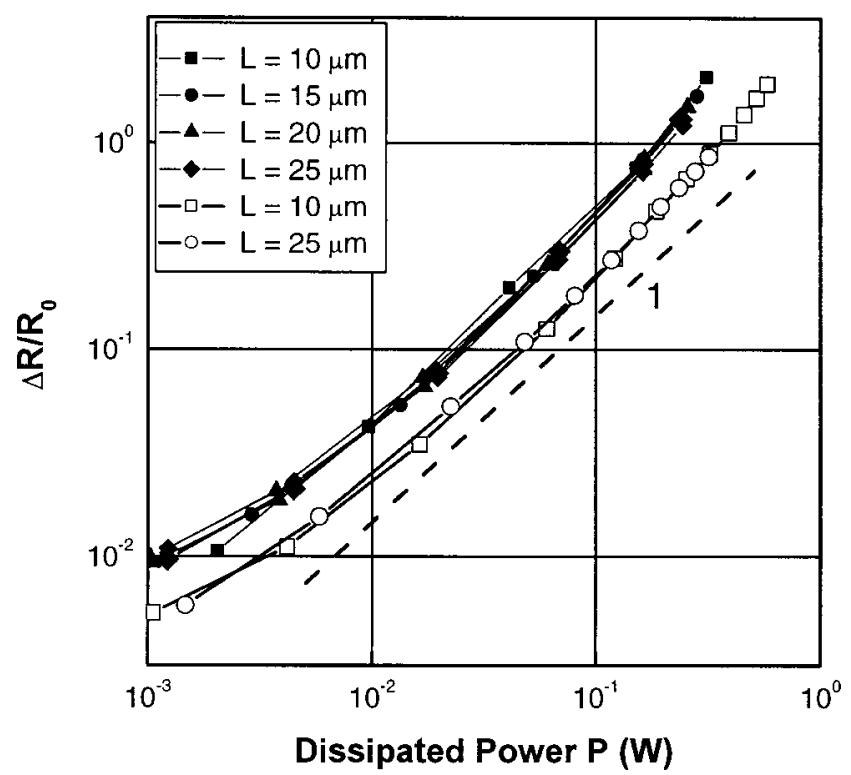

FIG. 6. The relative change of resistance of TLM structures of the sample of different lengths measured as a function of dissipated power: under conditions of active cooling (temperature kept constant at $290 \mathrm{~K}$ ) —empty symbols and without active cooling at room temperature-filled symbols. Lengths of the samples are shown in inset.

two and more times with applied voltage) and samples have different lengths it is convenient to investigate noise and transport characteristics as a function of dissipated power $P(P=I V)$. To compare changes in the resistance of samples of different lengths we will analyze a relative increase of the resistance $\Delta R / R_{0}$ (where $R_{0}$ is the resistance measured at an applied voltage that corresponds to the linear regime in the CVC). The dependence of relative resistance $\Delta R / R_{0}$ on dissipated power for samples of different length is shown in Fig. 6. The measurements were carried out in two regimes: with and without active heat sink, which maintains the temperature of the sample at $290 \mathrm{~K}$. In both regimes, under high dissipated power that corresponds to the presence of nonequilibrium noise $\left(P>10^{-3} \mathrm{~W}\right)$, the dependence is almost linear. Taking into account this result and the fact that the curves for samples of different lengths coincide we conclude that the main reason for the resistance change is purely thermal. Relative changes in magnitude of $\Delta R / R_{0}$ under different cooling regimes can be explained by different degrees of the overheating effect.

At low temperatures the linear behavior of the dependence is conserved. The scaling coefficient of the dependence is determined by the conditions of thermal conductivity and heat dissipation elements of the system. The variable part of the resistance $[\Delta R$ in (2)] contributes to the total resistance and, consequently, to its fluctuations only when high voltages are applied. Therefore, fluctuations of $\Delta R$ are nonequilibrium fluctuations when the sample bias corresponds to the nonlinear part in the $I(\mathrm{~V})$ curve. The dependence of these fluctuations on dissipated power is shown in Fig. 7. The slope of the dependence $f S_{I} / I^{2}(0)=F(P)$ as well as the slope of the dependence $\Delta R / R_{0}=f(P)$ are equal to unity. Moreover, the amplitudes of nonequilibrium noise measured as a function of dissipated power for different

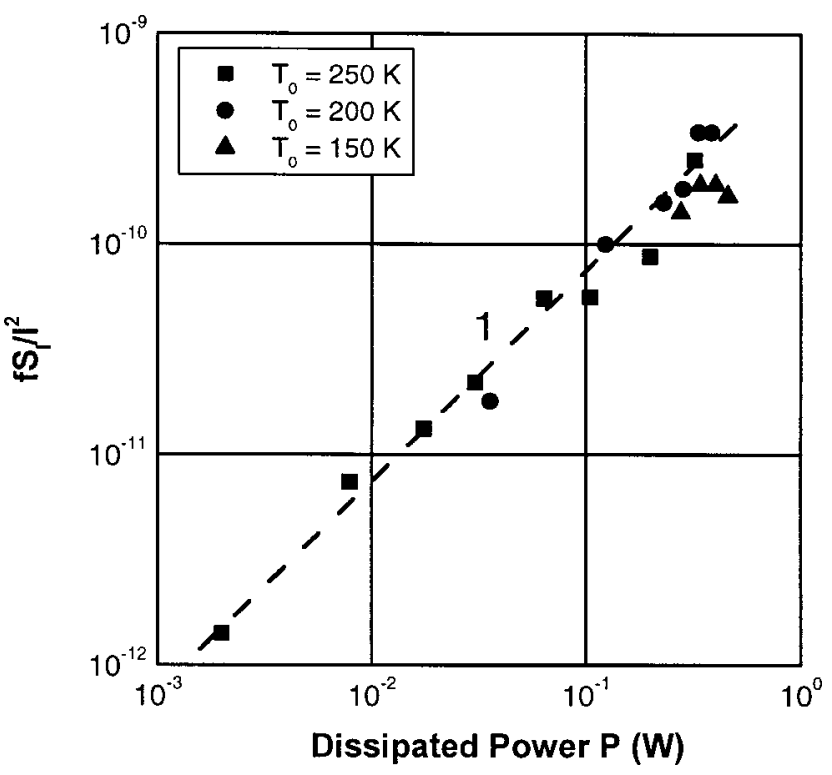

FIG. 7. Normalized spectral density of nonequilibrium part of $1 / f$ noise vs dissipated power plotted for minimum analyzed frequency of $1 \mathrm{~Hz}$. Sample length is equal to $25 \mathrm{~mm}$. The sample temperatures are shown in the inset.

sample temperatures coincide. Thus, the amplitude of nonequilibrium fluctuations of sample resistance depends only on value of dissipated power and does not depend on the absolute temperature of the sample. Therefore, nonequilibrium fluctuations can be caused either by an applied voltage (as part of dissipated power) or by overheating of the sample due to self-heating effects. It should be pointed out that the amplitude of nonequilibrium fluctuations is saturated with increasing applied voltage and the shorter the sample the lower the saturation value.

Additional information about the origin of the nonequilibrium processes in the structures can be obtained from the analysis of the frequency at which the transition from $1 / f$ noise to $1 / f^{2}$ noise is observed, i.e., transient frequency of nonequilibrium noise versus dissipated power. The dependence shown in Fig. 6 have well developed exponential behavior. It is seen in Fig. 8 that the transient frequency strongly depends on the sample temperature. Thus thermal activation is the most appropriate mechanism of relaxation of the fluctuations.

As we observed elsewhere, ${ }^{15}$ a low-frequency $1 / f$ noise and high-frequency $1 / f$ noise are correlated. The noise level $S_{I} / I^{2}$ of the high- frequency noise does not change with applied voltage. Therefore, we could suggest that lowfrequency (the nonequilibrium $1 / f$ ) noise represents by itself an enhanced high-frequency (equilibrium $1 / f$ ) noise. Moreover, the amplification occurs via a mechanism possessing frequency dispersion with particular time that corresponds to observed transient frequency.

\section{Proposed model of nonequilibrium noise}

The results obtained allow us to propose the following model of nonequilibrium oscillations in gateless TLM devices. Under equilibrium conditions in the conducting channel, equilibrium fluctuations of conductivity result in the usually observed equilibrium $1 / f$ noise. By increasing the 


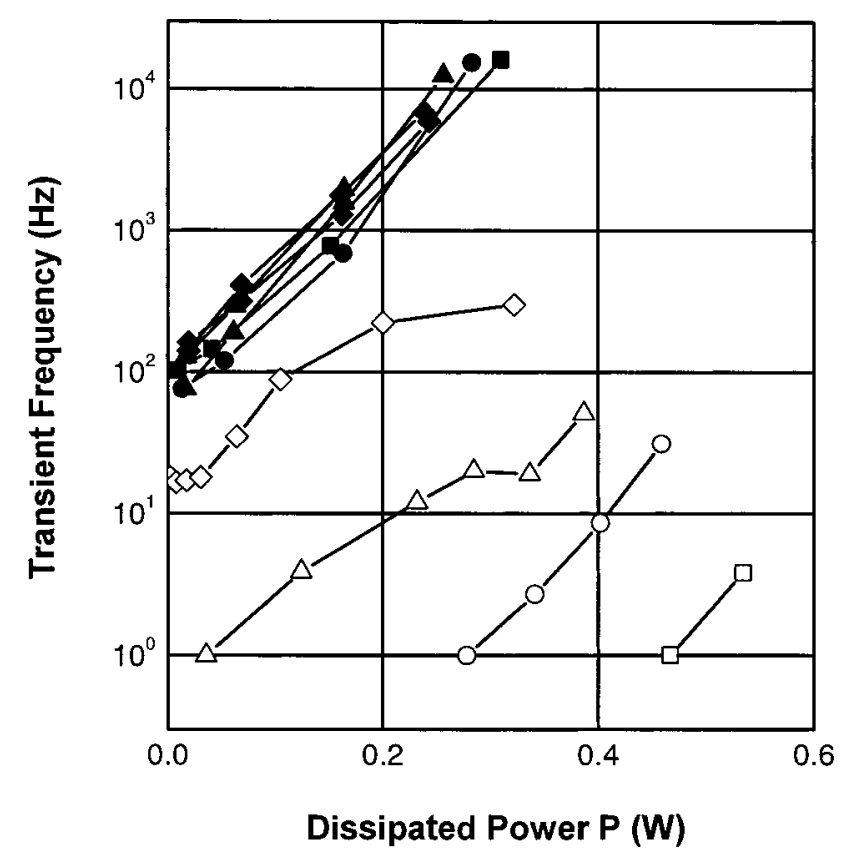

FIG. 8. The transient frequency dependence on dissipated power: (a) filled symbols; samples of different lengths $\mathrm{L}$ without cooling: squares- $10 \mu \mathrm{m}$, circle $-15 \mu \mathrm{m}$, triangles $-20 \mu \mathrm{m}$, diamonds $-25 \mu \mathrm{m}$; (b) open symbols; (samples with $L=25 \mu \mathrm{m}$ measured at different temperatures $T_{0}$ : squares$100 \mathrm{~K}$, circles- $150 \mathrm{~K}$, triangles-200 K, diamond-250 K.

voltage (at a sufficiently high applied voltage bias) at any moment in time a momentary nonuniform distribution of resistance in the sample along the current line is formed. The latter causes the formation of high-field and low-field regions in the channel, respectively. In high-field regions self-heating effects are stronger and lead to a further increase of local resistance and, consequently, to amplification of fluctuations. This process cannot be infinite and amplification of fluctuations is limited due to an increase of heat removal with temperature difference between the channel and substrate. Indeed, such a saturation was registered experimentally.

Obviously, amplification of fluctuations should occur at a rate determined by the time constant of a self-heating effect. At the same time, the characteristic times obtained from the noise measurements are significantly longer than heat relaxation times estimated from our experiments. ${ }^{3}$ Therefore, another factor determining the transient frequency should be considered. A more probable mechanism is coupling between surface and channel systems, which leads to redistribution of electron density along the channel under applied voltage. ${ }^{4}$

In our $\mathrm{AlGaN} / \mathrm{GaN}$ heterostructures 2D-electron gas (2DEG) is formed without any doping, only due to piezoelectric and spontaneous polarization effects. ${ }^{24}$ The polarization (positive) charges prevent instantaneous redistribution of potential along the channel and fast formation of the local self-heating in the channel. Therefore, the $1 / f$ noise amplification is expected to appear only in the frequency range below the transient frequency determined by the relaxation time of positive charge fluctuations. The time that is necessary to restore electrostatic equilibrium has activation behavior (Fig. 6). This time is longer than free carriers drift time and should be related to the electron emission from the traps in the barrier. At frequencies higher than the transient frequency electrostatic equilibrium cannot be restored and electrons captured by traps in the barrier move by hops. As a result an additional channel responsible for nonequilibrium noise appears. It represents by itself a hopping conductivity with thermal activation of carriers to the conducting channel.

\section{CONCLUSIONS}

In summary, we investigated the fluctuation phenomena in 2DEG in gateless $\mathrm{AlGaN} / \mathrm{GaN}$ TLM heterostructures at different electric fields and temperatures. The results of measurements at low voltages reveal the usual $1 / f$ noise. The Hooge coefficient was determined as $\alpha_{H}=4 \times 10^{-4}$, which agrees fairly well with previously reported data. At voltages higher than $1 \mathrm{~V}$ deviations from $1 / f$ noise were observed. The pronounced transformation of the spectra is a clear sign of nonequilibrium noise behavior. The amplitude of nonequilibrium fluctuations of the sample resistance only depends on the value of dissipated power and does not show any dependence on the absolute temperature of a sample. The features were analyzed together with nonlinearity effects on the dc current-voltage characteristic and can be ascribed to nonequilibrium effects induced by lateral electrical field redistribution.

\section{ACKNOWLEDGMENTS}

This work was supported by the Office of Naval Research under Grant No. N00014-01-1-0828 (Project Monitor Dr. Colin Wood) and Deutsche Forschungsgemeinschaft (Project No. KL 1342).

${ }^{1}$ U. K. Mishra and P. Parikh, Proc. IEEE 90, 1022 (2002).

${ }^{2}$ A. Balandin, S. V. Morozov, S. Cai, R. Li, K. L. Wang, G. Wijeratne, and C. R. Viswanathan, IEEE Trans. Microwave Theory Tech. 47, 1413 (1999).

${ }^{3}$ S. A. Vitusevich et al., Appl. Phys. Lett. 82, 748 (2003).

${ }^{4}$ B. K. Ridley, J. Appl. Phys. 90, 6135 (2001).

${ }^{5}$ Z. C. Huang, R. Goldberg, J. C. Chen, Y. Zheng, D. B. Mott, and P. Shu, Appl. Phys. Lett. 67, 2825 (1995).

${ }^{6}$ T. H. Yu and K. F. Brennan, J. Appl. Phys. 91, 3730 (2002).

${ }^{7}$ N. Balkan, M. C. Arikan, S. Goleden, V. Tilak, B. Shaff, and R. J. Shealy, J. Phys.: Condens. Matter 14, 3457 (2002).

${ }^{8}$ R. F. Voss and J. Clarke, Phys. Rev. Lett. 36, 42 (1976).

${ }^{9}$ H. G. Beck and W. P. Spruit, J. Appl. Phys. 49, 3384 (1978).

${ }^{10}$ R. F. Voss and J. Clarke, Phys. Rev. B 13, 556 (1976).

${ }^{11}$ S. M. Bezrukov and M. Winterhalter, Phys. Rev. Lett. 85, 202 (2000).

${ }^{12}$ I. V. Krive, E. N. Bogachek, A. G. Scherbakov, and U. Landman, Phys. Rev. B 64, 233304 (2001).

${ }^{13}$ M. Sampierto, G. Ferrari, and G. Bertuccio, J. Appl. Phys. 87, 7583 (2000).

${ }^{14}$ G. P. Zhigal'skii, Usp. Fiz. Nauk 173, 465 (2003).

${ }^{15}$ S. A. Vitusevich et al., Proceedings of ICPS26, Edinburg, UK, 2002.

${ }^{16}$ J. A. Garrido, B. E. Foutz, J. A. Smart, J. R. Murphy, W. J. Schaff, and L. F. Eastman, Appl. Phys. Lett. 76, 3442 (2000).

${ }^{17}$ A. L. McWhorter, in Semiconductor surface Physics, edited by R. H. Kingston (University of Philadelphia Press, Philadelphia, PA, 1957), pp. 207-228.

${ }^{18}$ F. N. Hooge, T. G. H. Kleinpenning, and L. K. J. Vandamme, Rep. Prog. Phys. 44, 479 (1981).

${ }^{19} \mathrm{M}$. Tacano et al., Proceedings of 17 th International Conference on Noise and Fluctuations, August 18-22, 2003, Prague, Czech Republic, pp. 117122 . 
${ }^{20} \mathrm{~A}$. Van der Ziel, Noise in solid state devices and circuits (Wiley, New York, 1986).

${ }^{21}$ M. Shulz, J. Appl. Phys. 74, 2649 (1993).

${ }^{22}$ H. H. Mueller, D. Woerle, and M. Schulz, J. Appl. Phys. 75, 2970 (1994).
${ }^{23}$ T. A. Fulton and G. J. Dolan, Phys. Rev. Lett. 59, 109 (1987).

${ }^{24}$ O. Ambacher, B. Foutz, J. Smart, J. R. Shealy, N. J. Weimann, K. Chu, M. Murphy, A. J. Sierakowski, W. J. Schaff, and L. F. Eastman, J. Appl. Phys. 87, 334 (2000). 